\title{
PARESTESIAS ASSOCIADAS COM PROCEDIMENTOS ODONTOLÓGICOS: UMA REVISÃO INTEGRATIVA DE LITERATURA ${ }^{1}$
}

\author{
PARESTHESIA ASSOCIATED WITH DENTISTRY PROCEDURES: \\ AN INTEGRATIVE LITERATURE REVIEW
}

\author{
Júlia Tavares Palmeira², Samara Crislâny Araújo de Sousa ${ }^{3}$, \\ Quemuel Pereira da Silva ${ }^{4}$ e Jorge Pontual Waked ${ }^{5}$
}

\section{RESUMO}

O objetivo do presente estudo foi revisar a literatura odontológica para verificar quais os tipos de procedimentos mais associados à parestesia. Trata-se de uma revisão integrativa da literatura, constituída de 8 artigos. A busca dos estudos foi realizada nas bases de dados PubMed e Scielo utilizando os seguintes descritores, de forma isolada ou combinada: parestesias, complicações intra-operatórias, ensaios clínicos em Odontologia. Os critérios de inclusão foram artigos publicados nos últimos 10 anos (2010 a 2020), em língua portuguesa ou inglesa, que estivessem disponíveis na íntegra, publicados e indexados nas referidas bases de dados. Os critérios de exclusão foram artigos com disponibilidade apenas do resumo, monografias, dissertações, teses e editoriais. A literatura consultada mostrou que a maior ocorrência de parestesias provenientes de procedimentos odontológicos está associada ao uso de anestésico local (37,5\%), seguida de exodontias de terceiros molares $(25 \%)$, tratamento endodôntico (12,5\%), implante dentário (12,5\%) e osteotomia sagital bilateral (12,5\%). Os procedimentos mais relacionados à parestesia foram a administração de anestésico local, exodontias de terceiros molares, tratamento endodôntico, implante dentário e osteotomia sagital bilateral. Para que a ocorrência destas complicações seja evitada, é importante que o profissional elabore um devido planejamento de caso antes da execução, isso inclui a utilização de todo o aparato diagnóstico existente, como exames imaginológicos, clínicos e laboratoriais, por exemplo.

Palavras-chave: Condução nervosa, Sensação, Complicações intraoperatórias.

\section{ABSTRACT}

The objective of the presente study was to review the dental literatura to verify which types of dental procedures are most associated with paresthesia. This is an integrative literature review, consisting of 8 articles. The search for the studies was carried out in the PubMed and Scielo databases using the following descriptors, either alone or in combination: paresthesia, intra-operative complications and dentistry clinical trials. The inclusion criteria were articles published in the last 10 years (2010 to 2020), in Portuguese or English, which were available in full, published and indexed in those databases. Exclusion criteria were articles with availability only of the abstract, academic dissertations and editorials. The consulted literature showed that

1 Revisão Integrativa de Literatura.

2 Graduanda em Odontologia, Centro de Saúde e Tecnologia Rural, Universidade Federal de Campina Grande (UFCG), Patos - PB, Brasil. E-mail: julia.palmeira@hotmail.com

3 Graduanda em Odontologia, Centro de Saúde e Tecnologia Rural, Universidade Federal de Campina Grande (UFCG), Patos - PB, Brasil. E-mail: samaracrislany06@gmail.com

4 Cirurgião-Dentista Centro de Saúde e Tecnologia Rural, Universidade Federal de Campina Grande (UFCG), Patos PB, Brasil. E-mail: quemuelpereira7@gmail.com

5 Professor Dr. Adjunto do Curso de Odontologia, Centro de Saúde e Tecnologia Rural, Universidade Federal de Campina Grande (UFCG), Patos - PB, Brasil. E-mail: jpwaked@hotmail.com 
the grater occurrence of paresthesias from dental procedures is associated with the use of local anesthetic (37,5\%), followed by extractions of third molars (25\%), endodontics treatment $(12,5 \%)$, dental implant (12,5\%) and bilateral sagital osteotomy (12,5\%). The procedures most related to paresthesia were administration of local anesthetic, extractions of third molars, endodontics treatment, dental implant and bilateral sagittal osteotomy. For the occurrence of these complications to be avoided, it is important that the professional elaborate a proper case planning before the execution, this includes the use of all the existing diagnostic apparatus, such as imaging, clinical and laboratory exams, for example.

Keywords: Neural conduction, Sensation, Intraoperative complications.

\section{INTRODUÇÃO}

A parestesia é definida como uma neuropatia com alteração de sensações e anestesia persistente. Os sintomas relacionados à parestesia podem ser múltiplos e incluem função sensorial prejudicada, como anestesia ou hiperestesia, e perturbações neurossensoriais. (PICCINNI et al., 2015)

$\mathrm{Na}$ Odontologia, as parestesias faciais se manifestam frequentemente por meio dos nervos alveolar inferior, lingual, mentual, trigêmeo e facial, e são decorrentes de fatores locais ou sistêmicos. Dentre as possíveis causas, as relacionadas a problemas endodônticos merecem destaque, visto que a relação anatômica estabelecida entre os ápices radiculares e alguns feixes nervosos é muito próxima. (ALVES et al., 2016)

De acordo com Alves et al. (2014), a parestesia tem variadas causas: 1) mecânicas: traumas físicos aos nervos como compressão, estiramento ou ruptura (parcial ou total); 2) patológicas: tumores cujo crescimento provoca a compressão de nervos da região, acarretando danos às fibras nervosas sensitivas e consequente prejuízo sensorial; 3) físicas: excesso de calor, como no caso da realização de osteotomia com instrumentos rotatórios sob inadequada refrigeração; 4) químicas: aplicação de anestésicos locais e/ou outras substâncias nas imediações do nervo; 5) microbiológicas: infecções em tecidos moles ou duros.

As lesões traumáticas em nervos periféricos podem ser classificadas em lesões do tipo neuropraxia, axonotmese e neurotmese, em função da intensidade do comprometimento das estruturas do nervo e a suas manifestações clínicas. Em adição, Sunderland classificou as lesões em cinco grupos nos quais a lesão de primeiro grau corresponde à neuropraxia, do segundo ao quarto grau é a axonotmese, subdividida em ordem progressiva, e a de quinto é equivalente à neurotmese. (KIM, 2016)

A neuropraxia consiste em uma interrupção transitória da condução nervosa na qual o axônio permanece íntegro e a sua recuperação é espontânea, no entanto pode levar semanas ou até meses. A axonotmese ocorre quando o axônio dentro do nervo é rompido, porém o epineuro permanece preservado. Nesta situação, ocorre o fenômeno da degeneração walleriana, quando há degradação da parte distal do axônio juntamente com a perda das células de Schwann. Já a neurotmese é classificada como o 
pior tipo de lesão, quando há a secção total do nervo. Nesta situação, raramente a recuperação ocorre espontaneamente, exigindo intervenção cirúrgica para obter melhores resultados. (JONES et al., 2016)

Existem várias consequências do dano neural, em região perioral e oral, que vão desde déficit sensorial, como anestesia (perda completa de sensação) ou hipoestesia (sensibilidade diminuída a todas as formas de estimulação), a distúrbios neuro-sensoriais anormais tais como parestesia (sensação de entorpecimento, sensação de ardor ou picada), hiperestesia (sensibilidade aumentada) e alodinia (dor por estímulo que normalmente não é doloroso quando aplicado em outro lugar do corpo). Quando o nervo lingual está envolvido, o ramo corda do tímpano do nervo facial também tem a possibilidade de ser afetado, levando a disgeusia (alteração do paladar) e xerostomia (salivação reduzida). (CHAN et al., 2010)

Existem maiores chances de danos ao nervo alveolar inferior após as exodontias de terceiros molares inferiores com impactação óssea total, quando comparados aos terceiros molares totalmente erupcionados. A impactação horizontal também é associada com comprometimento ao nervo alveolar inferior (NAI) (FLORES et al., 2011). As parestesias podem também ser provocadas devido a outros procedimentos odontológicos, como é o caso da neurotoxicidade causada pelas soluções anestésicas de articaína 4\% e de prilocaína 3\% (GARISTO et al., 2010).

Dependendo da etiologia da parestesia, o tratamento desta pode ser realizado através de acupuntura, laserterapia, tratamento medicamentoso, microneurocirurgia, eletroestimulação, fisioterapia ou calor úmido (CASTRO, 2015).

O objetivo do presente estudo foi revisar a literatura odontológica para verificar quais tipos de procedimentos odontológicos estão mais susceptíveis a gerar parestesia em ambulatório odontológico.

\section{METODOLOGIA}

Esse estudo refere-se a uma revisão integrativa da literatura sobre procedimentos odontológicos associados à parestesia, na qual a busca dos artigos foi desenvolvida de forma virtual em periódicos cadastrados nas bases de dados PubMed e Scielo, utilizando os seguintes descritores de forma isolada ou combinada: parestesia, complicações intraoperatórias e odontologia. Os critérios de inclusão foram artigos publicados nos últimos 10 anos (2010 a 2020), em língua portuguesa ou inglesa, que estivessem disponíveis na íntegra, publicados e indexados nas referidas bases de dados. Os critérios de exclusão foram artigos com disponibilidade apenas do resumo, monografias, dissertações, teses e editoriais.

A análise e a confecção dos resultados encontrados nos estudos incluídos nesta revisão foram feitas de modo descritivo, em tabela, com o objetivo de organizar e compilar os principais dados relativos aos estudos. A tabela contempla os seguintes itens: título do artigo, autor principal, ano de publicação, tamanho da amostra, periódico onde o artigo foi publicado, objetivo e resultados. 


\section{RESULTADOS}

A busca dos artigos foi realizada por dois pesquisadores, no período de fevereiro a abril de 2021, sendo selecionadas as publicações que atendiam aos critérios de inclusão. Em relação aos países de publicação dos artigos, houve três artigos (37,5\%) no Brasil, um (12,5\%) na Turquia, um (12,5\%) na República Tcheca, um (12,5\%) na Coréia do Sul e um $(12,5 \%)$ no Canadá.

Foram identificados 8 estudos que relatam a ocorrência de parestesia em procedimentos odontológicos (Tabela 1)

Tabela 1 - Detalhamento de casos de parestesias em procedimentos odontológicos.

\begin{tabular}{|c|c|c|c|c|c|}
\hline $\mathbf{N}^{\mathbf{o}}$ & Título do Artigo & Autor / Ano & $\begin{array}{c}\text { Amostra/Informações } \\
\text { do estudo }\end{array}$ & Objetivo & Resultados \\
\hline 1 & $\begin{array}{l}\text { Avaliação comparativa } \\
\text { de articaína } 4 \% \text { versus } \\
\text { mepivacaína } 2 \% \text { para } \\
\text { cirurgia de terceiros } \\
\text { molares: ensaio clínico } \\
\text { controlado, randomiza- } \\
\text { do, duplo-cego, de boca } \\
\text { dividida }\end{array}$ & Almeida (2019) & $\begin{array}{l}\text { Estudo clínico controla- } \\
\text { do de boca dividida de } \\
20 \text { pacientes com tercei- } \\
\text { ros molares inferiores } \\
\text { bilaterais em posições } \\
\text { semelhantes, dividido } \\
\text { em dois grupos, com } \\
\text { mepivacaína e articaína, } \\
\text { respectivamente. }\end{array}$ & $\begin{array}{l}\text { Foram avaliados o uso } \\
\text { dos anestésicos locais } \\
\text { cloridrato de articaína } \\
4 \% \text { e do cloridrato de } \\
\text { mepivacaína } 2 \% \text {, em } \\
\text { exodontias de terceiros } \\
\text { molares inferiores. }\end{array}$ & $\begin{array}{l}\text { A ocorrência de parestesia } \\
\text { foi observada com os dois } \\
\text { tipos de anestésicos, sendo } \\
3 \text { do grupo de articaína e } 1 \\
\text { caso no grupo da mepiva- } \\
\text { caína, todas reversíveis em } \\
30 \text { dias. }\end{array}$ \\
\hline 2 & $\begin{array}{l}2 \text { mm é uma distância } \\
\text { segura do nervo alveo- } \\
\text { lar inferior para evitar } \\
\text { complicações neuros- } \\
\text { sensoriais na cirurgia } \\
\text { de implante? }\end{array}$ & $\begin{array}{l}\text { Tufekcioglu } \\
\text { et al. (2017) }\end{array}$ & $\begin{array}{l}\text { Estudo de coorte } \\
\text { retrospectivo. Foram } \\
\text { avaliados } 474 \text { implan- } \\
\text { tes, em } 314 \text { pacientes, } \\
\text { posicionados posterior- } \\
\text { mente à área do forame } \\
\text { mentual }\end{array}$ & $\begin{array}{l}\text { Comparar as compli- } \\
\text { cações neurossenso- } \\
\text { riais relacionadas aos } \\
\text { implantes inseridos } \\
\text { a menos de } 2 \mathrm{~mm} \text { do } \\
\text { nervo alveolar inferior } \\
\text { (NAI) com as inseridas } \\
\text { a mais de } 2 \mathrm{~mm}\end{array}$ & $\begin{array}{l}153 \text { implantes }(32,2 \%) \\
\text { foram inseridos a menos } \\
\text { de } 2 \mathrm{~mm} \text { do NAI enquan- } \\
\text { to } 321 \text { implantes }(67,8 \%) \\
\text { a mais de } 2 \mathrm{~mm} \text {. Três } \\
\text { implantes com distância } \\
\text { de } 0 \text { mm ao NAI }(0,63 \%) \\
\text { causaram parestesia após } \\
\text { a cirurgia. A distância do } \\
\text { implante não produziu } \\
\text { diferença estatística signi- } \\
\text { ficativa em relação à dor e } \\
\text { à parestesia. }\end{array}$ \\
\hline 3 & $\begin{array}{l}\text { Prevalência de pares- } \\
\text { tesia decorrente do } \\
\text { tratamento endodôn- } \\
\text { tico no município de } \\
\text { João Pessoa-PB }\end{array}$ & $\begin{array}{l}\text { Veloso et al. } \\
\text { (2017) }\end{array}$ & $\begin{array}{l}\text { Foi um estudo de preva- } \\
\text { lência, descritivo, } \\
\text { quantitativo, transversal } \\
\text { com } 48 \text { endodontistas } \\
\text { cadastrados no CRO }\end{array}$ & $\begin{array}{l}\text { Avaliar a prevalência } \\
\text { de parestesia decorren- } \\
\text { te do tratamento endo- } \\
\text { dôntico no município } \\
\text { de João Pessoa-PB. }\end{array}$ & $\begin{array}{l}31,3 \% \text { dos entrevistados } \\
\text { apresentaram casos de } \\
\text { parestesia. Destes, } 57,9 \% \\
\text { citaram como fator etio- } \\
\text { lógico a anestesia e } 81,3 \% \\
\text { chegaram ao diagnóstico } \\
\text { através da queixa de } \\
\text { dormência prolongada pelo } \\
\text { paciente. O nervo alveolar } \\
\text { inferior e o mentual foram } \\
\text { os mais citados, com } 60 \% \\
\text { e } 33,3 \% \text { respectivamente. }\end{array}$ \\
\hline
\end{tabular}




\begin{tabular}{|c|c|c|c|c|c|}
\hline 4 & $\begin{array}{l}\text { Parestesia após anesté- } \\
\text { sicos locais: uma análi- } \\
\text { se de relatórios para o } \\
\text { sistema de notificação } \\
\text { de eventos adversos } \\
\text { da FDA }\end{array}$ & $\begin{array}{l}\text { Piccinni et al. } \\
\text { (2015) }\end{array}$ & $\begin{array}{l}\text { Estudo descritivo e ana- } \\
\text { lítico com } 528 \text { relatos } \\
\text { de Parestesias obtidos } \\
\text { no Sistema de Relatório } \\
\text { de Eventos Adversos da } \\
\text { FDA (FAERS) }\end{array}$ & $\begin{array}{l}\text { Avaliar os possíveis si- } \\
\text { nais de alerta de pares- } \\
\text { tesia pelos anestésicos } \\
\text { locais, enfocando os } \\
\text { usados em odontologia }\end{array}$ & $\begin{array}{l}\text { A análise dos dados cole- } \\
\text { tados apontou uma maior } \\
\text { relação entre os casos de } \\
\text { parestesia com o uso da ar- } \\
\text { ticaina } 4 \% \text { e o segundo mais } \\
\text { prevalente foi a prilocaina. }\end{array}$ \\
\hline 5 & $\begin{array}{l}\text { Acidentes e compli- } \\
\text { cações associados a } \\
\text { cirurgias de terceiros } \\
\text { molares realizadas por } \\
\text { estudantes de odonto- } \\
\text { logia. }\end{array}$ & $\begin{array}{l}\text { Azenha et al. } \\
\text { (2013) }\end{array}$ & $\begin{array}{l}\text { Estudo retrospectivo } \\
\text { de } 122 \text { prontuários de } \\
\text { pacientes submetidos } \\
\text { a cirurgias de terceiros } \\
\text { molares }\end{array}$ & $\begin{array}{l}\text { Demonstrar as taxas de } \\
\text { acidentes e complica- } \\
\text { ções em cirurgias de } \\
\text { terceiros molares reali- } \\
\text { zadas por estudantes de } \\
\text { Odontologia }\end{array}$ & $\begin{array}{l}\text { Os casos de acidentes e } \\
\text { complicações totalizaram } \\
10,4 \% \text {, sendo } 0,9 \% \text { os de } \\
\text { parestesia. }\end{array}$ \\
\hline 6 & $\begin{array}{l}\text { O papel do posiciona- } \\
\text { mento intraoperatório do } \\
\text { nervo alveolar inferior } \\
\text { na parestesia pós-ope- } \\
\text { ratória após osteotomia } \\
\text { sagital bilateral da man- } \\
\text { díbula: estudo clínico } \\
\text { prospectivo. }\end{array}$ & $\begin{array}{c}\text { Hanzelka et al. } \\
\text { (2011) }\end{array}$ & $\begin{array}{l}\text { Estudo prospectivo em } \\
290 \text { pacientes sub- } \\
\text { metidos à osteotomia } \\
\text { sagital bilateral }\end{array}$ & $\begin{array}{l}\text { O objetivo deste estudo } \\
\text { foi avaliar a relação } \\
\text { entre procedimentos de } \\
\text { osteotomia sagital bila- } \\
\text { teral da mandíbula com } \\
\text { parestesia do nervo } \\
\text { alveolar inferior }\end{array}$ & $\begin{array}{l}\text { A parestesia se desenvol- } \\
\text { veu imediatamente após a } \\
\text { cirurgia em quase metade } \\
\text { dos pacientes e a maioria } \\
\text { dos casos foi resolvido } \\
\text { dentro de } 1 \text { ano após a } \\
\text { cirurgia. }\end{array}$ \\
\hline 7 & $\begin{array}{l}\text { Integridade cortical do } \\
\text { canal alveolar inferior } \\
\text { como preditor de pares- } \\
\text { tesia após extração do } \\
\text { terceiro molar. }\end{array}$ & $\begin{array}{l}\text { Park et al. } \\
\text { (2010) }\end{array}$ & $\begin{array}{l}\text { Estudo de coorte } \\
\text { retrospectivo com } 179 \\
\text { participantes subme- } \\
\text { tidos à extração de } \\
\text { terceiro molar inferior }\end{array}$ & $\begin{array}{l}\text { Avaliar a relação entre } \\
\text { parestesia após extra- } \\
\text { ção do terceiro molar } \\
\text { inferior e a integridade } \\
\text { cortical do canal al- } \\
\text { veolar inferior usando } \\
\text { tomografia computado- } \\
\text { rizada }\end{array}$ & $\begin{array}{l}\text { A prevalência geral de pa- } \\
\text { restesia foi de } 4,2 \% \text { ( } 11 \text { de } \\
259 \text { dentes). O resultado do } \\
\text { estudo afirma que a perda } \\
\text { da integridade cortical } \\
\text { está associada a um risco } \\
\text { aumentado de parestesia } \\
\text { após a extração. }\end{array}$ \\
\hline 8 & $\begin{array}{l}\text { Ocorrência de pareste- } \\
\text { sia após administração } \\
\text { de anestésico local } \\
\text { odontológico nos Esta- } \\
\text { dos Unidos }\end{array}$ & $\begin{array}{l}\text { Garisto et al. } \\
\qquad(2010)\end{array}$ & $\begin{array}{l}\text { Estudo descritivo e ana- } \\
\text { lítico com } 248 \text { relatos } \\
\text { do Evento Adverso da } \\
\text { Food and Drug Ad- } \\
\text { ministration dos EUA } \\
\text { (Sistema de relatórios) }\end{array}$ & $\begin{array}{l}\text { Determinar se o tipo } \\
\text { de anestésico local ad- } \\
\text { ministrado teve algum } \\
\text { efeito nos relatos de } \\
\text { parestesia em pacientes } \\
\text { odontológicos nos Esta- } \\
\text { dos Unidos. }\end{array}$ & $\begin{array}{l}248 \text { casos de parestesia } \\
\text { foram relatados, } 94,5 \% \text { en- } \\
\text { volvendo bloqueio do nervo } \\
\text { mandibular e } 89 \% \text { o nervo } \\
\text { lingual de forma individual } \\
\text { e concomitante. }\end{array}$ \\
\hline
\end{tabular}

\section{DISCUSSÃO}

Na prática odontológica é frequente encontrar casos de parestesia em pacientes que foram submetidos a exodontias, consideravelmente em terceiros molares inferiores ou cirurgias que envolvam a proximidade de feixes vásculo-nervosos. Na região mandibular, a mais frequentemente afetada pelas parestesias, estruturas nobres como o nervo lingual, o nervo alveolar inferior e o nervo bucal estão presentes (GRILLO, 2010). No caso de uma intervenção nessa área, um toque ou uma manipulação indesejada no feixe nervoso poderá correr o risco de desencadear uma parestesia (ZOTELLI, 2010). Corroborando com os estudos acima, foi possível avaliar que os procedimentos odontológicos que estão mais passíveis de ocorrer parestesia são: anestesia local (37,5\%), exodontia de terceiros molares (25\%), implante (12,5\%), tratamento endodôntico (12,5\%) e osteotomia sagital bilateral $(12,5 \%)$. 
Embora os anestésicos locais sejam seguros, eficazes e medicamentos essenciais para a Odontologia, o uso destes poderá causar complicações. Um destes resultados é a parestesia, que se refere a uma neuropatia que se manifesta como anestesia persistente ou uma sensação alterada variando de dormência completa a queimação, formigamento ou dor contínua (GARISTO et al., 2010). Por existirem estudos (ALMEIDA, 2019; GARISTO et al., 2010; PICCINNI et al., 2015) que relacionam o anestésico local (37,5\% da amostra total) e a ocorrência de uma parestesia, é necessário que haja outros estudos mais robustos que tenham a capacidade de confirmar a ocorrência de parestesia a partir da neurotoxicidade dos sais anestésicos, visto que a utilização dessa substância é frequentemente utilizada na maioria dos procedimentos odontológicos.

Os indivíduos buscam atendimento odontológico com o propósito de resolver problemas funcionais e estéticos, contudo, mesmo procedimentos odontológicos considerados simples podem causar parestesia. A parestesia pode afetar negativamente a qualidade de vida dos pacientes interferindo na mastigação, além de promover acúmulo de restos alimentares sobre a mucosa jugal, mordidas frequentes nos lábios e queimaduras com líquidos quentes (FLORES et al., 2011) (RENTON, 2010). O presente estudo reforça o quão se torna necessária a correta utilização dos métodos de diagnóstico complementares. Os exames imaginológicos são excelentes ferramentas que, além de auxiliarem o diagnóstico, também podem fornecer informações que possibilitam a prevenção de complicações associadas às parestesias (AZENHA et al., 2014).

Previamente a qualquer procedimento cirúrgico o paciente deve ser informado dos possíveis acidentes e/ou complicações que possam ocorrer durante o tratamento, estando ciente de que toda e qualquer situação não planejada deverá ser tratada da maneira mais adequada possível. A forma mais adequada de prevenção das situações inesperadas é o planejamento do procedimento cirúrgico (AZENHA et al., 2014).

Quando ocorre a secção do nervo, as técnicas de microneurocirurgia poderão ser usadas a fim de restabelecer a função sensorial ou motora. Essas técnicas propostas possuem um número pouco expressivo de casos nos quais se obteve êxito, caracterizando a parestesia como uma condição de improvável resolutividade na maioria das situações, principalmente naquelas relacionadas com o nervo lingual (FLORIAN, 2011).

\section{CONCLUSÃO}

Os procedimentos mais relacionados à parestesia foram a administração de anestésico local, exodontias de terceiros molares, tratamento endodôntico, implante dentário e osteotomia sagital bilateral. Para que a ocorrência destas complicações seja evitada, é importante que o profissional elabore um devido planejamento de caso antes da execução, isso inclui a utilização de todo o aparato diagnóstico existente, como exames imaginológicos, clínicos e laboratoriais, por exemplo. 


\section{REFERÊNCIAS}

ALVES, A. L. R. F. A. et al. Parestesia, por que o endodontista deve se preocupar Full Dentistry In Science, v. 29, n. 8, p. 133-140, 2016.

ALVES, F. R.; COUTINHO, M. S.; GONÇALVES, L. S. Endodontic-Related Facial Paresthesia: systematic review. Journal Of The Canadian Dental Association, v. 80, n. 13, p. 1-7, 2014.

AZENHA, M. R. et al. Accidents and complications associated to third molar surgeries performed by dentistry students. Oral Maxillofacial Surgery, v. 18, n. 4, p. 459-464, 2014.

CASTRO, A. L. F. Tratamento da parestesia do nervo alveolar inferior e lingual no pós operatório de $3^{\circ}$ molar: revisão de literatura. Revista Científica Cro, v. 2, n. 16, p. 34-42, 2015.

CHAN, H. L. et al. The Significance of the Lingual Nerve During Periodontal/Implant Surgery. Journal Of Periodontology, v. 81, n. 3, p. 372-377, 2010.

FLORES, F. W. et al. Parestesia do nervo alveolar inferior após exodontia de terceiros molares inferiores inclusos. International Journal of Dentistry, v. 10, n. 4, p. 268-273, 2011.

FLORIAN, M. R. Disfunção temporomandibular e acupuntura: uma terapia integrativa e complementar. Odontologia Clínico-Científica, v. 2, n. 10, p. 189-192, 2011.

GARISTO, G. A. et al. Occurrence of paresthesia after dental local anesthetic administration in the United States. The Journal Of The American Dental Association, v. 141, n. 7, p. 836-844, 2010.

GRILLO, C. M. Tratamento da disfunção temporomandibular com acupuntura: relato de caso clínico. Revista Paulista de Odontologia, v. 1, n. 32, p. 31-33, 2010.

HANZELKA, T. The role of intraoperative positioning of the inferior alveolar nerve on postoperative paresthesia after bilateral sagittal split osteotomy of the mandible: prospective clinical study. International Journal Of Oral \& Maxillofacial Surgery, v. 9, n. 40, p. 901-906, 2011.

JONES, S.; EINSENBERG, H.M.; JIA, X. Advances and Future Applications of Augmented Peripheral Nerve Regeneration. International Journal of Molecular Sciences, v. 17, n. 9, p. 1-9, 2016. 
KIM, J. Neural Reanimation Advances and New Technologies. Facial Plastic Surgery Clinics of North America, v. 24, n. 1, p. 71-84, 2016.

PARK, W. et al. (2010). Cortical Integrity of the Inferior Alveolar Canal as a Predictor of Paresthesia After Third-Molar Extraction. The Journal Of The American Dental Association, v. 141, n. 3, p. $271-278,2010$.

PICCINNI, C. et al. Paraesthesia after local anaesthetics: an analysis of reports to the FDA Adverse Event Reporting System. Basic \& Clinical Pharmacology Toxicology, v. 117, n. 1, p. 52-56, 2015.

RENTON, T. Prevention of Iatrogenic Inferior Alveolar Nerve Injuries in Relation to Dental Procedures. Dental Update, v. 37, n. 6, p. 350-363, 2010.

TUFEKCIOGLU, S. Is $2 \mathrm{~mm}$ a safe distance from the inferior alveolar canal to avoid neurosensory complications in implant surgery? Niger Journal Clinical Practice, v. 20, n. 3, p. 274-277, 2017.

VELOSO, H. H. P. Prevalência de parestesia decorrente do tratamento endodôntico no município de João Pessoa-PB. Revista Odontológica do Brasil Central, v. 26, n. 79, p. 42-47, 2017.

ZOTELLI, V. L. R. Uso da acupuntura no manejo da dor em pacientes com alterações na articulação temporomandibular (ATM). Revista Odontológica da Unicid, v. 22, n. 2, p. 185-188, 2010. 